\title{
ON DEGENERATE FULLY NONLINEAR ELLIPTIC EQUATIONS IN BALLS
}

\author{
NEIL S. TRUDingER
}

We establish derivative estimates and existence theorems for the Dixichlet and Neumann problems for nonlinear, degenerate elliptic equations of the form $F\left(D^{2} u\right)=g$ in balls. The degeneracy. arises through the possible vanishing of the function $g$ and the degenerate Monge-Ampère equation is covered as a special case.

This note concerns boundary value problems of Dirichlet and Neumann type for degenerate nonlinear elliptic equations of the form.

$$
F\left(D^{2} u\right)=g \text {, }
$$

where the function $F$ is similar to those treated by Caffarelli, Nirenberg and spruck [2]. The degeneracy arises through the vanishing of the non-negative function $g$. Here we shall restrict attention to balls in Euclidean $n$ space, $\mathbb{R}^{n}$, making use of an argument which originated in [10], but hopefully this special case will shed some light on the general situation. The results here also improve previous work on the elliptic Monge-Ampère equation,

$$
\operatorname{det} D^{2} u=g
$$

Received 30 April 1986.

Copyright Clearance Centre, Inc. Serial-fee code: 0004-9729/87 $\$ A 2.00+0.00$. 
but again, we stress, only in balls. The Dirichlet problem for (2) has received considerable attention in recent years ([1], [4], [5], [6], [8], [11]). The Neumann problem was recently treated by Lions, Trudinger and Urbas [10].

To formulate appropriate conditions on $F$, we let $\$^{n}$ denote the linear space of $n \times n$ real symetric matrices and suppose that $F$ is defined on an open convex cone $\Gamma \subset \$^{n}$, with vertex at the origin and containing the cone of positive matrices. We then assume

$F \in C^{O}(\bar{\Gamma}) \cap C^{2}(\Gamma)$ satisfies:

(i) $F$ is positive on $\Gamma$ and vanishes on $\partial \Gamma$,

(ii) $F$ is elliptic on $\Gamma$ which means $F^{\prime}$ is positive there,

(iii) $E$ is concave on $\Gamma$,

(iv) $F(x) \rightarrow \infty$ as $r \rightarrow \infty$, (that is, as the minimum eigenvalue $\rightarrow \infty)$.

Further hypotheses will be invoked as needed. For the Monge-Ampere equation, we note that these hypotheses are fulfilled when $\Gamma$ is the cone of positive matrices and

$$
F(r)=(\operatorname{det} r)^{1 / n} \text {. }
$$

In conjunction with equation (1) in the unit ball $B$ in Euclidean $n$ space $\mathbb{R}^{n}$, we consider Dirichlet boundary conditions,

$$
u=\phi \text { on } \partial B \text {, }
$$

and Neumann boundary conditions of the form

$$
\frac{\partial u}{\partial r}+\gamma u=\psi \text { on } \partial B,
$$

where $\phi, \gamma, \psi$ are functions defined on $\partial B$. Through the method of continuity, the solvability of these problems for sufficiently smooth $g, \phi, \gamma, \psi$ depends upon a priori estimates of solutions and their first and second derivatives which we now establish. Throughout we shall assume that a solution $u$ of equation (1) is admissible in the sense that $D^{2} u \in \bar{\Gamma}$. 


\section{Solution estimates}

For these we may consider any sufficiently smooth domain $\Omega$ in $\mathbb{R}^{n}$ and a boundary condition of the form,

$$
B \cdot D u+r u=\psi \text { on } \partial \Omega
$$

where $\beta \cdot \nu \geq 0, \gamma \geq \gamma_{0}, \nu$ denotes the unit outer normal to $\partial \Omega$ and $\gamma_{0}$ is a positive constant. Taking $0 \in \Omega$ and setting $w(x)=A|x|^{2}$ we can by virtue of (iv) make $F\left(D^{2} w\right)$ arbitrarily large by taking .the constant $A$ sufficiently large. Fixing $A$ so that

$$
F\left(D^{2} w\right) \geq \sup _{\Omega} g \text {, }
$$

it then follows from the comparison principle that

$$
\frac{1}{\gamma_{0}}\left(\text { inf } \psi-2 A \sup _{\partial \Omega}|\beta| \operatorname{diam} \Omega\right) \leq u \leq \frac{1}{\gamma_{0}} \sup _{\partial \Omega} \phi .
$$

First derivative estimates

For the Dirichlet problem, these are elementary and essentially covered by [2]. Differentiating (1), we obtain for $k=1, \ldots, n$,

$$
E^{i j}\left(D^{2} u\right) D_{i j k} u=D_{k} g
$$

where $\left[F^{i j}\right]=F^{\prime}$. Now, using the concavity of $F$, we have

$$
F\left(D^{2} w\right)-F\left(D^{2} u\right) \leq F^{i j}\left(D^{2} u\right) D_{i j}(w-u)
$$

so that

$$
\begin{aligned}
F^{i j}\left(D^{2} u\right) D_{i j} w & \geq F\left(D^{2} w\right)-F\left(D^{2} u\right)+F^{i j}\left(D^{2} u\right) D_{i j} u \\
& \geq F\left(D^{2} w\right)-g
\end{aligned}
$$

by virtue again of the concavity of $F$ and equation (1). With the constant $A$ again chosen sufficiently large, we obtain for any domain $\Omega$,

$$
\sup _{\Omega}|D u| \leq \sup _{\partial \Omega}|D u|+C|g|_{1 ; \Omega}
$$

But then $\sup _{\partial B}|D u|$ is readily bounded with the aid of barrier functions 
of the form

$$
w^{ \pm}= \pm A\left(1-|x|^{2}\right)+\phi
$$

As a result we obtain,

$$
\sup _{B}|D u| \leq C\left(|g|_{1}+|\phi|_{2}\right)
$$

where $C$ depends on $n$ and trace $F^{\prime}(I)$. A first derivative estimate for the Neumann problem (5) will be deduced by interpolation of the second derivative estimate.

\section{Second derivative estimates}

We set

$$
v=\Delta u-x_{i} x_{j} D_{i j} u
$$

noting that on $\partial B$,

$$
v=\Delta_{\partial B} u+(n-1) \frac{\partial u}{\partial r},
$$

where $\Delta_{\partial B}$ is the Laplace Beltrami operator in $S^{n-1}$. By computation, as in $[10]$, we have

$$
\begin{aligned}
& F^{i j} D_{i j} v=\Delta g-x_{i} x_{j} D_{i j} g-F^{i j, p q}\left(\delta_{k \ell}-x_{k} x_{\ell}\right) D_{i j k} u D_{p q \ell} u \\
& \text { - } 2 F^{i j} D_{i}\left(x_{k} x_{\ell}\right) D_{j k \ell} u-F^{i j} D_{i j}\left(x_{k} x_{\ell}\right) D_{k \ell} u
\end{aligned}
$$

where

$$
F^{i j, p q}(a)=\frac{\partial^{2} F}{\partial a_{i j} \partial a_{p q}}
$$

for any $a=\left[a_{i j}\right] \in \Phi^{n}$. Consequently using the concavity of $F$ and (8),

$$
\begin{aligned}
F^{i j} D_{i j} v & \geq \Delta g-x_{i} x_{j} D_{i j} g-4 x_{i} D_{i} g-2 F^{i j} D_{i j} u \\
& \geq \Delta g-x_{i} x_{j} D_{i j} g-4 r \frac{\partial g}{\partial r}-2 g .
\end{aligned}
$$

Hence if the Dirichlet boundary condition (4) is satisfied, we get using (9), (11) 
(16)

$$
\begin{aligned}
\sup _{B} v & \leq \sup _{\partial B} v+C|g|_{2 ; B} \\
& \leq C\left(|\phi|_{2 ; \partial B}+|g|_{2 ; B}\right)
\end{aligned}
$$

where $C$ depends only on $n$ and trace $F^{\prime}(I)$. Now if we impose a further condition on $\Gamma$, namely

$$
a \leq(\text { trace } a) I
$$

for any $a \in \Gamma$, we deduce from (16) the interior estimate

$$
\left|D^{2} u(x)\right| \leq \frac{C}{1-|x|}\left(|\phi|_{2 ; \partial B}+|g|_{2 ; B}\right)
$$

where $C$ depends only on $n$. Note that the dependence on trace $F^{\prime}(I)$ from (12) disappears since (17) implies trace $a \geq 0$, whence $u$ is subharmonic. For the Monge Armpère equation (2), the estimate (18) extends the interior second derivative estimates of Pogorelov [11] and Trudinger and Urbas [13] to non-negative inhomogeneous terms $g$ (but only in balls!). Next suppose that the Neumann boundary condition (5) is satisfied. By computation we then obtain on $\partial B$,

$$
\begin{aligned}
\frac{\partial v}{\partial r} & =\left(\delta_{i j}-x_{i} x_{j}\right) D_{i j}\left(\frac{\partial u}{\partial r}\right)-2 \Delta u \\
& =\Delta_{\partial B}\left(\frac{\partial u}{\partial r}\right)-2 \Delta u+(n-1) \frac{\partial^{2} u}{\partial r^{2}}, \\
& =\Delta_{\partial B}(\psi-\gamma u)-2 \Delta u+(n-1) \frac{\partial^{2} u}{\partial r^{2}},
\end{aligned}
$$

so that combining (15) and (19), we get an estimate

$$
v \leq C\left[|g|_{2 ; B}+|\psi|_{2 ; B}+|u|_{1 ; B}+\sup _{\partial B}\left|\frac{\partial^{2} u}{\partial r^{2}}\right|\right]
$$

where $C$ depends on $n$ and $|\gamma|_{2 ; B}$. To estimate $\frac{\partial^{2} u}{\partial r^{2}}$, we now compute

$$
F^{i j} D_{i j}\left(x_{k} D_{k} u-\gamma u\right)=2 F^{i j} D_{i j} u+x_{k} D_{k} g-F^{i j} D_{i j}(\gamma u)
$$

so that with barrier considerations similar to (11), we obtain 


$$
\sup _{\partial B}\left|\frac{\partial^{2} u}{\partial r^{2}}\right| \leq C\left[|g|_{1 ; B}+|\psi|_{2 ; B}+|u|_{1 ; B}\right]
$$

and hence conbining (20) and (22), we have

$$
\sup _{\partial B} \Delta u \leq C\left[|g|_{1 ; B}+|\psi|_{2 ; B}+|u|_{1 ; B}\right] \text {. }
$$

But since

$$
F^{i j} D_{i j} \Delta u \geq \Delta g \text { in } B
$$

we can infer a global bound

$$
\sup _{B} \Delta u \leq C\left[|g|_{1 ; B}+|\psi|_{2 ; B}+|u|_{1 ; B}\right] \text {. }
$$

Using (25) and the concavity of $F$, we then obtain the full second derivative bound,

$$
\sup _{B}\left|D^{2} u\right| \leq C\left[|g|_{2 ; B}+|\psi|_{2 ; \partial B}+|u|_{1 ; B}\right]
$$

where $C$ depends on $n,|\gamma|_{2 ; \partial B}$ and $F^{\prime}(I)$, and finally by interpolation,

$$
|u|_{2 ; B} \leq C\left[|g|_{2 ; B}+|\psi|_{2 ; \partial B}\right]
$$

\section{Existence theorems.}

The second derivative estimates (18) and (27) can now be used to solve the corresponding boundary value problems through approximation by nondegenerate problems. In the case of the Dirichlet problem we replace the function $g$ by $g+\epsilon$ for positive $\epsilon$ and approximate the boundary value $\phi$ by $C^{4}(\partial B)$ functions. If the functions $F$ are further restricted to fulfill all the conditions of Caffarelli, Nirenberg and Spruck [2], the approximating Dirichlet problems can be solved classically. In particular for their prime examples, the elementary symetric functions of the eigenvalues of $D^{2} u$,

$$
F_{k}\left(D^{2} u\right)=\left\{\sum_{i_{1}<\ldots<i_{k}} \lambda_{i_{1}} \ldots \lambda_{i_{k}}\right\}^{1 / k}, k=1, \ldots, n,
$$


the cone $\Gamma$ is the component of the set where $F_{k}$ is positive which contains the positive cone. We thus conclude from the estimates (7), (12), (18) and [2, Theorem 3]:

THEOREM 1. Let $g \geq 0, \in c^{1,1}(\bar{B}), \phi \in c^{1,1}(\partial B)$. Then there exists a unique solution $u \in C^{0,1}(\bar{B}) \cap C^{1,1}(B)$ with $D^{2} u \subset \bar{\Gamma}$ of the Dirichlet problems,

$$
F_{k}\left(D^{2} u\right)=g \text { in } B, u=\phi \text { on } \partial B
$$

Observe that we can in particular allow $g$ to vanish everywhere in $B$. In the Monge-Ampere case, this was proved in [13] while the case when $\phi=0, g \neq 0$ was established by Chen [3].

For Neumann boundary conditions (5) the results are somewhat stronger as we need only assume $F$ satisfies conditions (i) to (iv). Here we again replace $g$ by $g+\epsilon$ for positive $\epsilon$ so the approximating equations become uniformly elliptic with respect to solutions with bounded second derivatives. Invoking the second derivative Hölder estimates of Lions and Trudinger [9], (see also [7], [12]) we can then establish the classical solvability of the approximating boundary value problems. Thus we obtain the following.

THEOREM 2. Let $F$ satisfy (i) to (iv) with respect to some cone $r$, $g \geq 0, \epsilon C^{1,1}(\bar{B}), \gamma, \psi \in C^{1,1}(\partial B)$ with $\gamma \geq \gamma_{0}$ for some positive constont $\gamma_{0}$. Then there exists a unique solution $u \in C^{1,1}(\bar{B})$ with $D^{2} u \subset \bar{\Gamma}$ of the boundary value problem,

$$
F\left(D^{2} u\right)=g \text { in } B, \quad \frac{\partial u}{\partial r}+\gamma u=\psi \text { on } \partial B .
$$

It is plausible to conjecture that Theorem 1 and 2 extend at least from balls to sufficiently smooth uniformly convex domains but even in the Monge Ampère case, this is an open question.

\section{References}

[1] L. Caffarelli, L. Nirenberg, J. Spruck, "The Dirichlet problem for nonlinear second order order elliptic equations I. Monge-Ampère equation", Carm. Prore Appl. Math. 37 (1984), 369-402. 
L2」 L. Caffarelli, L. Nirenberg, J. Spruck, "The Dirichlet problem for nonlinear second order elliptic equations, III. Functions of the eigenvalues of the Hessian", Acta Math. 155 (1985), 261-301.

[3] Y. Chen "Degenerate Monge-Ampère equations" (to appear.

[4] S.-Y. Cheng, S.-T. Yau, "On the regularity of the Monge-Ampère equation $\operatorname{det}\left(\partial^{2} u / \partial x_{i} \partial x_{j}\right)=F(x, u) "$, Comm. Pure App $z$. Math. 30 (1977), 41-68.

[5] N. M. Ivochkina, "An a priori estimate of $\|u\| C^{2}(\bar{\Omega})$ for convex solutions of the Dirichlet problem for the Monge-Amperre equations", Zep. Naucn. Sem. Leningrad. Otdez. Mat. Inst. Steklov. (LOMI) 96 (1980), 69-79 [Russian]. English translation in J. Soviet Math. 21 (1983), 689-697.

[6] N. V. Krylov, "On degenerate nonlinear elliptic and parabolic equations in a domain", Izv. Akad. Nauk. SSSR 47 (1983), 75-108, [Russian].

[7] G. M. Lieberman, N. S. Trudinger, "Nonlinear oblique boundary value problems for nonlinear elliptic equations", Trans. Amer. Math. Soc. 295 (1986), 509-546.

[8] P.-L. Lions, "Sur les equations de Monge-Ampere I", Mconuscripta Math. 41 (1983), 1-44.

[9] P.-L. Lions, N. S. Trudinger, "Linear oblique derivative problems for the uniformly elliptic Hamilton-Jacobi-Bellman equation", Math. 2. 191 (1986), 1-15.

[10] P.-L. Lions, N. S. Trudinger, J. I. E. Urbas, "The Neumann problem for equations of Monge-Ampère type", Comm. Pure Appl. Math. 39 (1986), 539-563.

[11] A. V. Pogorelov, "The Dirichlet problem for the $n$-dimensional analogue of the Monge-Ampère equation", Dokz. Akad. Nouk SSSR 201 (1971), 790-793, [Russian]. English translation in Soviet Math. Dok2. 12(1971), 1727-1731.

[12] N. S. Trudinger, "Boundary value problems for fully nonlinear elliptic equations", Proc. Centre for Math. Anal. Aust. Nat. univ. 8 (1984), 65-83.

[13] N. S. Trudinger, J. I. E. Urbas, "On second derivative estimates for equations of Monge-Ampère type", Bulz. Austral. Math. Soc. 30 (1983), 321-334. 
Centre for Mathematical Analysis

Australian National University

GPO Box 4

Canberra ACT 2601

AUSTRALIA. 